\title{
Light- and Electron-Microscopical Study of Phosphoprotein B-50 Following Denervation and Reinnervation of the Rat Soleus Muscle
}

\author{
J. Verhaagen, ${ }^{1,2}$ A. B. Oestreicher, ${ }^{2}$ P. M. Edwards, ${ }^{2}$ H. Veldman, ${ }^{1}$ F. G. I. Jennekens, ${ }^{1}$ and W. H. Gispen ${ }^{2}$ \\ 'Department of Neurology, University Hospital, 3500 CG Utrecht, and 'Division of Molecular Neurobiology, Rudolf Magnus \\ Institute for Pharmacology and Institute of Molecular Biology and Medical Biotechnology, University of Utrecht, $3584 \mathrm{CH}$ \\ Utrecht, The Netherlands
}

\begin{abstract}
The neuron-specific phosphoprotein B-50 was originally identified as a phosphoprotein in synaptic plasma membranes isolated from adult brain tissue. In this paper we study the reinnervation of the soleus muscle, a target muscle of sciatic nerve axons, using affinity-purified anti-B-50 antibodies. Light-microscopical evaluation of the reinnervation process revealed that the period of muscle fiber reinnervation corresponds closely with the time in which high B-50 immunoreactivity was observed in the nerve fibers that invade the muscle and in the newly formed neuromuscular junctions. Upon completion of reinnervation, B-50 immunoreactivity decreased. In the newly innervating terminals, B-50 was associated with presynaptic vesicular structures and with the presynaptic plasma membrane. In intact mature neuromuscular junctions, virtually no B-50 immunoreactivity could be detected with either light- or electron-microscopic procedures. These observations corroborate the association of high levels of B-50/GAP43 during axon outgrowth and support the concept that B-50 may be a key molecule in the reconstruction of axonal structures. We also observed an unexpected transient increase in B-50 immunoreactivity in the degenerating neuromuscular junctions. This observation cannot be explained in terms of increased neuronal synthesis of B-50, since the degenerating axon processes have been completely disconnected from their cell bodies. Thus, our evidence implies that a rise of B-50 immunoreactivity can be associated with stages of neuronal degeneration as well as with those of neuronal differentiation and axon outgrowth.
\end{abstract}

The morphological and biochemical changes observed in a nerve following nerve damage have been widely studied. Much is known concerning the morphological changes that accompany the processes of degeneration and eventual regeneration of a damaged nerve. The specific molecular events that underlie these morphological changes remained largely unknown. A major approach to the evaluation of biochemical correlates of the regeneration response has been the analysis by 1 - and 2-dimensional gel electrophoresis of pulse-labeled neuronal proteins that are

\footnotetext{
Received June 22, 1987; revised Sept. 29, 1987; accepted Sept. 29, 1987

The authors thank Ed Kluis for preparing the figures and Marie-Jeanne MensMeert and Lis Claessens for their excellent secretarial assistance.

Correspondence should be addressed to W. H. Gispen, Division of Molccular Neurobiology, Rudolf Magnus Institute for Pharmacology and Institute of Molecular Biology and Medical Biotechnology, University of Utrecht, Padualaan 8, $3584 \mathrm{CH}$ Utrecht, The Netherlands.

Copyright (C) 1988 Society for Neuroscience $0270-6474 / 88 / 051759-08 \$ 02.00 / 0$
}

conveyed to the damaged axon profiles by axoplasmic transport. The rationale behind these experiments is that if a protein serves a specific function in the reconstruction of axons, it would be labeled and transported at substantially elevated levels in damaged axons, comparcd to intact axons. Using this approach, Skene and Willard (1981a, b), Benowitz et al. (1981), and Benowitz and Lewis (1983) identified a small set of proteins, designated growth-associated proteins (GAPs), that has been implicated as being specifically involved in the axonal repair process. One of these proteins, GAP43, is identical to the neuron-specific protein kinase C substrate B-50 (Jacobson et al., 1986).

B-50 was originally identified as a phosphoprotein in synaptic plasma membranes prepared from adult brains (Zwiers et al., 1976, 1980). Neurochemical evidence suggests that the phospho-form of B-50 inhibits the conversion of phosphatidylinositol 4-phosphate (PIP) to phosphatidylinositol 4,5-biphosphate $\left(\mathrm{PIP}_{2}\right)$ in synaptic plasma membranes, probably by modulating the activity of the PIP kinase. This could constitute an important feedback regulation of the receptor-mediated breakdown of $\mathrm{PIP}_{2}$, yielding diacylglycerol (DG) and inositol triphosphate $\left(\mathrm{IP}_{3}\right)$ as second-messenger molecules (Gispen, 1986). B-50/GAP43 is a prominent phosphoprotein in growth cones, the terminal axon structures that perform a specialized function in axonal growth (De Graan et al., 1985; Katz et al., 1985; Meiri et al., 1986; Skene et al., 1986). The modulation of the PIP-kinase by the phospho-form of B-50 has also been shown to occur in growth cone membranes (Van Hooff et al., 1988). The association of increased levels of B-50 with growing axons demonstrated in regenerating rat peripheral nerves has been shown by immunohistochemical and radioimmunoassay techniques (Verhaagen et al., 1986). The 5-fold increase in B-50 concentration $6 \mathrm{~d}$ following a crush lesion is consistent with the results of metabolic labeling studies on GAP43 (Skene and Willard, 1981a, b). This further supports the evidence that B-50 and GAP43 are the same protein.

In this paper the reinnervation of the soleus muscle, one of the target muscles of the sciatic nerve axons, is evaluated by immunohistochemical and immunoelectron-microscopical methods using anti-B-50 antibodies. B-50-immunoreactive intramuscular axons were rare in undamaged animals. High B-50 immunoreactivity was found during the period of muscle fiber reinnervation and declined to control values in the neuromuscular terminals and innervating axonal branches upon completion of reinnervation. The B-50 present in newly formed terminals was associated with presynaptic vesicular structures and with the presynaptic plasma membrane. These results are consistent with the growth-associated role of B-50. We also docu- 
ment an unexpectedly large increase in the number of B-50immunoreactive neuromuscular junctions that are undergoing degeneration. This finding cannot be explained in the light of current data and the origin of the immunoreactivity is currently under investigation. We feel it is important to communicate our unexpected observations at this stage because this is the first report on accumulation of B-50/GAP43 in circumstances where neural degeneration as well as regeneration are occurring.

The results are discussed in the light of previous findings suggesting that B-50/GAP43 is a key molecule in events operative in processes associated with neuronal plasticity.

\section{Materials and Methods}

Surgical procedures. Female rats of an inbred Wistar strain (TNO, Zeist, Netherlands; body weight, $120-140 \mathrm{gm}$ ) were subjected to a bilateral crush lesion of the sciatic nerve $27 \mathrm{~mm}$ distal from the sciatic notch (De Koning et al., 1986). The proximal border of the 2.0-mm-wide crush lesion was marked with an epineural suture in order to enable reproducible dissection. Transection of the nerve and ligation of the distal and proximal nerve stump were also performed $27 \mathrm{~mm}$ distal to the sciatic notch.

Gel electrophoresis and immunoblotting. Six days following the surgery, rats were decapitated and the sciatic nerves were isolated. From the epineural suture subsequently distal portions $(5 \mathrm{~mm})$ of nerve were cut out. Analogous nerve pieces were dissected out from the intact nerves. Immediately following homogenization in a Potter-type ho-

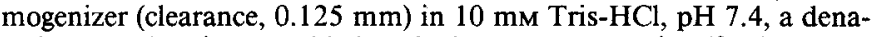
turing sample mix was added to the homogenate to give (final concentration): Tris- $\mathrm{HCl}, 62.5 \mathrm{~mm}, \mathrm{pH}$ 6.8; SDS, $1 \%$; glycerol, $10 \%$; bromophenol blue, $0.001 \%$; and 2-mercaptoethanol, $5 \%$. The homogenates were separated in $11 \%$ SDS-polyacrylamide gels (Zwiers et al., 1976) and protcins were transferred from the gels to nitrocellulose papers according to Towbin et al. (1979). The immune-incubation of blots with anti-B-50 antibodies has been previously described in detail (Oestreicher and Gispen, 1986).

Double-label immunohistochemistry. Muscles (m. soleus) were either fixed for $2.5 \mathrm{hr}$ in $2 \%$ paraformaldehyde $/ 50 \mathrm{~mm}$ phosphate buffer, $\mathrm{pH}$ 7.4 , containing $0.1 \mathrm{~m}$ lysine and $0.2 \%$ sodium periodate (PLP; McLean and Nakane, 1974), or immediately frozen in liquid nitrogen. The paraformaldehyde-fixed muscles were cryoprotected by immersion in graded sucrose up to a concentration of $35 \%$. Sections prepared from unfixed muscles were precipitation-fixed in $100 \%$ acetone for $20 \mathrm{~min}$. Prior to immunohistochemistry, muscle sections were stained for $\mathrm{AChE}$, as described by Pestronk and Drachman (1978). The double-labeling experiments were performed at room temperature, using mouse monoclonal anti-70 kDa ncurofilament (NF70) antibodies (Monosan, Leiden, Netherlands) and affinity-purified rabbit anti-B-50 antibodies (antiserum 8420). Cryostat sections, $8 \mu \mathrm{m}$ thick, were reacted for $24 \mathrm{hr}$ with a mixture of NF70 (dilution, 1:20) and B-50 (dilution, 1:800) antibodies (Verhaagen et al., 1986). The site of binding of anti-B-50 antibodies was visualized using rhodamine-conjugated swine anti-rabbit IgGs ( $1: 100$; Dako, Copenhagen, Denmark). Subsequently, the sections were incubated with a $1 \%$ normal rabbit IgG solution (Miles Lab., Merseyside, UK). Finally, fluorescein-conjugated rabbit anti-mouse antibodies $(1: 100$; Dako) were used to visualize the binding of anti-NF70 antibodies (Verhaagen et al., 1986). The sections were mounted in $0.1 \mathrm{M}$ 1.4-diazabicyclo- $(2,2,2)$-octane dissolved in $90 \%$ glycerol in veronal-buffered saline, $\mathrm{pH} 8.3$.

$A C h E$ silver-staining. Soleus muscles were dissected out and quickly frozen in liquid nitrogen-cooled isopentane. Longitudinal sections 50 $\mu \mathrm{m}$ thick were cut on a cryostat as follows. The superficial part of the muscle was cut away with the cryostat knife until the left and right tendons were in the plane of section. At that point every third section was collected. In this way, 3 sections per muscle were made available for histological examination. The sections were collected on formolgelatin-coated coverslips, which were made wet with a solution of $3 \%$ (wt/vol) EDTA to prevent muscle fiber contraction during thawing of the sections. The sections were air-dried with a dryer for $5 \mathrm{~min}$. AChE silver-staining was performed for $30-40 \mathrm{~min}$ at $37^{\circ} \mathrm{C}$, using the method of Pestronk and Drachman (1978). Upon light-microscopic inspection of the sections, the endplates are clearly visible as bright blue stained spots. Since denervation of muscle fibers results in a reduction in the intensity of the AChE staining, relatively long staining times (30-40 min) were employed. This ensured that denervated endplates were stained. In order to quantitate the number of innervated endplates, the scctions were examined in the light microscope at a magnification of $1000 \times$. When a silver-stained axon branch terminated in the bluestained endplate, this endplate was scored as "innervated." For each muscle, about 50 endplates (approximately 17 endplates/section) were screened for innervation.

Quantification of B-50-immunoreactive endplates. Soleus muscles were trimmed in the cryostat as described above. From the middle region of the muscle, $8-\mu \mathrm{m}$-thick longitudinal sections were collected on a formolgelatin-coated coverslip. From a series of 10 sections, only the first one was collected and examined. In this way, 10 sections per muscle were made available for histological examination. This sampling procedure prevents a single neuromuscular junction from being included in 2 sections. The sections were first stained for $\mathrm{AChE}$ and immunohistochemistry was then performed as follows. The sections were incubated for $24 \mathrm{hr}$ with affinity-purified anti-B-50 antibodies (1:800). Subsequently, the sections were rinsed with PBS/ $0.2 \%$ Triton X-100 and the site of antigen-antibody binding was visualized with fluorescein-conjugated swine anti-rabbit IgGs (1:100; Dako), as described above for the second-step antibodies in the section on double-label immunohistochemistry. The AChE-stained endplates that were also labeled with anti-B-50 antibodies (fluorescent endplates) were termed "B-50-immunoreactive" endplates.

Protein determination. Protein concentrations were measured using the method of Lowry et al. (1951).

Immunoelectron microscopy. Cryostat sections of 30- $\mu$ m-thick PLPfixed muscles were incubated for $8 \mathrm{hr}$ with affinity-purified anti-B-50 antibodies in PBS $/ 0.2 \%$ BSA (1:60). Sections were rinsed for $1 \mathrm{hr}$ with PBS and subsequently sections were incubated overnight with HRPconjugated swine anti-rabbit IgGs (Dako). Sections were stained for peroxidase according to the DAB (diaminobenzidine)-Ni-Co method of Adams (1981). The sections were postfixed in $1 \%$ osmium tetroxide for $60 \mathrm{~min}$ at room temperature, dehydrated, and embedded in Epon as in routine electron-microscopic procedures. Thin sections of muscles containing the endplate regions were contrasted with lead citrate for $1 \mathrm{~min}$ and subsequently visualized in the electron microscope.

\section{Results}

The binding of the affinity-purified rabbit anti-B-50 antibodies to proteins separated from nerve homogenates is illustrated in Figure 1. An immune band comigrating with B-50 from synaptosomal plasma membranes is present in immunoblots of nerve pieces prepared from a crushed sciatic nerve $6 \mathrm{~d}$ after crush. With the antibody dilutions that gave pronounced staining of B-50 in damaged nerve, no B-50 could be detected in control nerves. However, with higher antibody concentrations, low staining of a band at the height of B-50 could be secn, indicating that a small amount of $\mathrm{B}-50$ is present in intact nerves.

The reinnervation of the soleus muscle, one of the target muscles of sciatic nerve axons, was studied by AChE silverstaining. Figure 2 shows AChE silver-stained sections of soleus muscles at various days after nerve crush. Six days following the crush lesion of the sciatic nerve, all neuromuscular junctions (identified in the AChE-stained sections as bright blue spots) had lost their silver-stained axon profiles (Figs. 2, 4). The ingrowth of newly formed sprouts was monitored with the AChE silver-staining method. Counting of the number of reinnervated endplates shows that the first neuromuscular junctions are reinnervated between 8 and $12 \mathrm{~d}$ after crush. Between 18 and $31 \mathrm{~d}$, reinnervation is complete (Fig. $4 A$ ). Combined $\mathrm{AChE}$ immunohistochemical staining of sections prepared from mature control muscles revealed the presence of numerous intramuscular axons labeled with an anti-NF70 monoclonal antibody. In these control muscle sections, no B-50 immunoreactivity could be demonstrated in the neuromuscular junctions (Fig. 3). Furthermore, labeling of intramuscular axon bundles with anti-B-50 


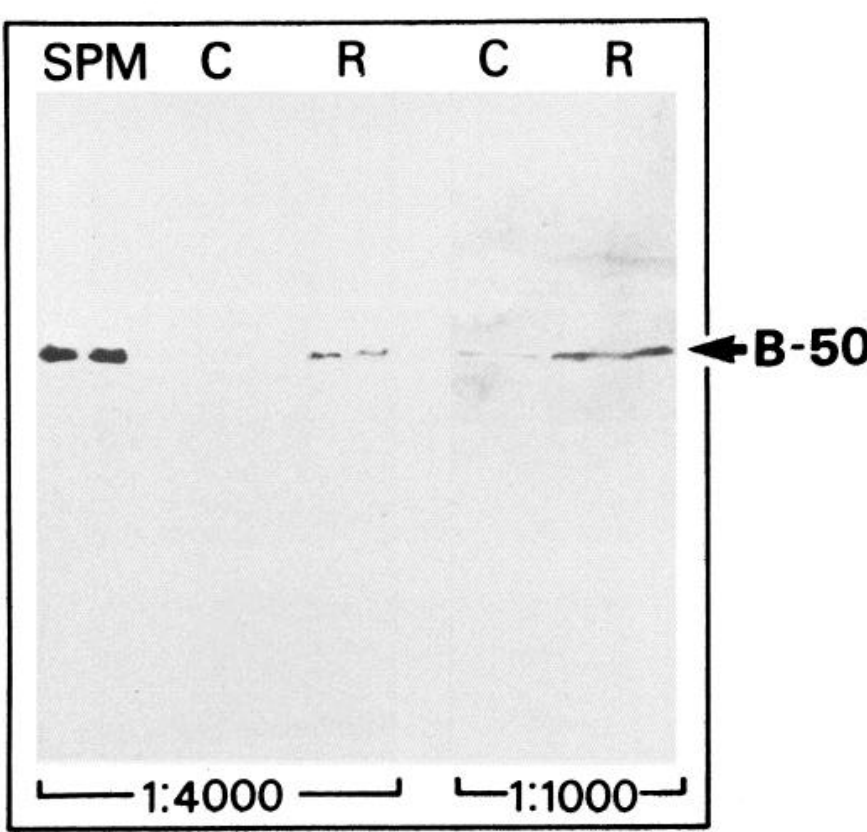

Figure 1. B-50 immunoreactivity on Western blots loaded with samples (40 $\mu \mathrm{g}$ protein/lane) obtained from intact control $(C)$ and crushed regenerating $(R)$ rat sciatic nerves. The anti-B-50 antibodies were used at dilutions of 1:4000 and 1:1000, as indicated. A light synaptosomal plasma membrane fraction (SPM; Kristjansson et al., 1982) was employed as a reference for B-50 localization on the immunoblot. Preimmune serum and secondary antibody controls were negative (data not shown). antibodies was virtually absent, although a small number of axons was clearly labeled in sections of mature, nondenervated muscles (Fig. 3). Three days after nerve crush, over $70 \%$ of neuromuscular junctions contained B-50 immunoreactivity (Fig. $4 B$, peak I), although at this time almost none contained silverstained axon profiles (Fig. 4A). Fewer B-50-immunoreactive neuromuscular junctions were observed $6 \mathrm{~d}$ following crush, but subsequently an increase in the percentage of B-50-immunoreactive junctions was obvious (Fig. $4 B$, peak II). Two months after the crush lesion, hardly any labeling was present in the muscle sections (Fig. $4 B$ ). Nerve transection resulted in the same initial increase in the number of B-50-immunoreactive neuromuscular junctions as was seen following a nerve crush, but under these experimental conditions no second peak in the number of labeled endplates was seen at day 18 , as had been found in muscles in which the sciatic nerve had been crushed. A comparison of the number of B-50-immunoreactive endplates in paraformaldehyde-fixed and acetone-treated sections revealed the presence of similar numbers of neuromuscular junctions under both conditions at various days following crush lesioning (data not shown).

Pre-embedding immunoelectron-microscopic examination of the localization of B-50 in reinnervated neuromuscular junctions $18 \mathrm{~d}$ after nerve crush revealed the presence of the immunoperoxidase precipitate on vesicular membranes and on the presynaptic plasma membrane of the nerve terminal (Fig. 5). Most of the labeled vesicles were of uniform size (about $50 \mathrm{~nm}$ ). Immunoreactivity was also observed in a few larger-type vesicular organelles (about 120-150 nm). In control endplates these structures were not labeled. Three days following nerve crush,

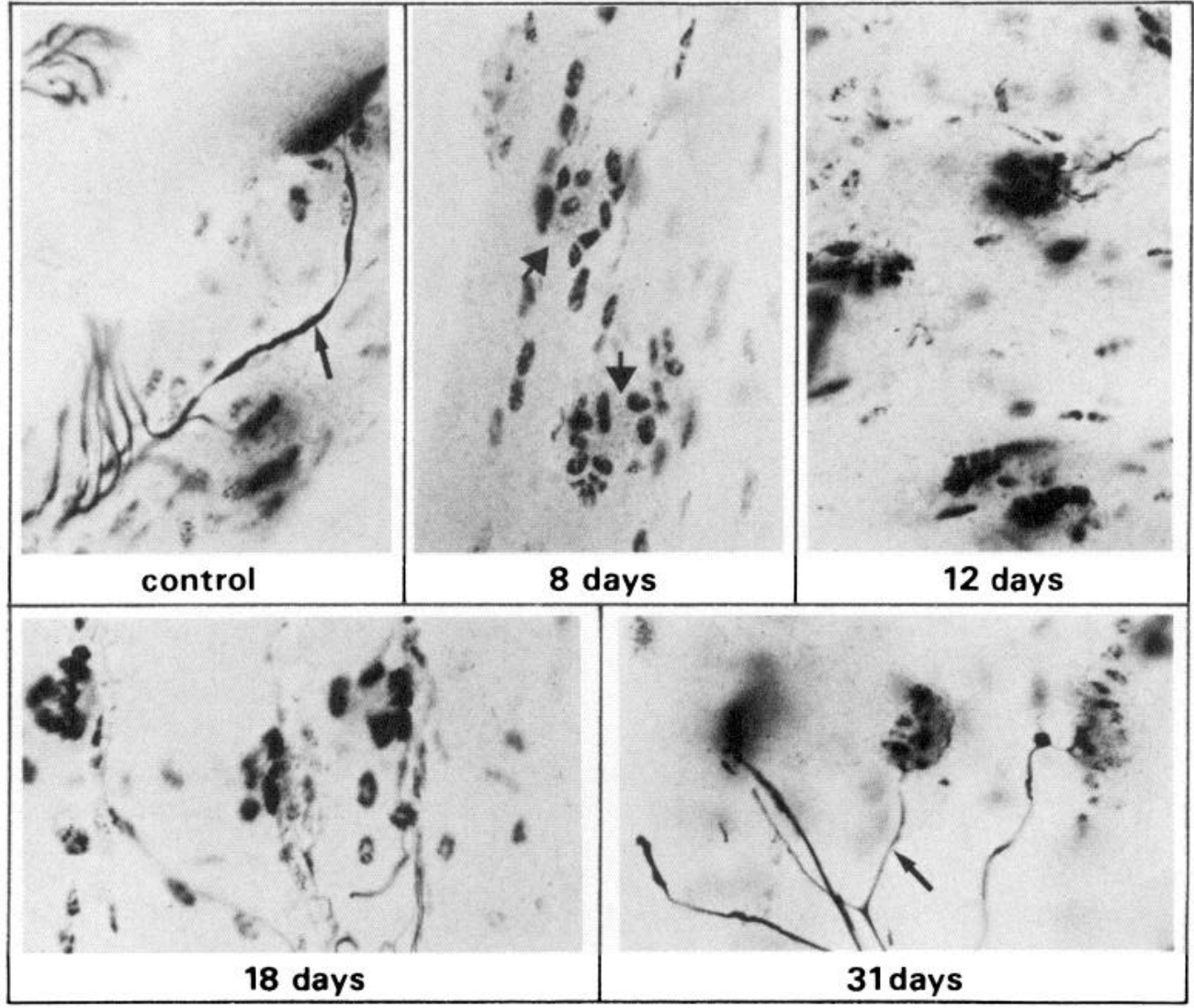

$\overline{20 \mu}$
Figure 2. AChE silver-stained, $50-\mu \mathrm{m}$ thick longitudinal sections of a control soleus muscle and of soleus muscles at $8,12,18$, and $31 \mathrm{~d}$ following crush denervation. AChE-stained areas (thick arrow) and silver-stained axon branches (thin arrows) can clearly be distinguished. 
Figure 3. AChE-stained longitudinal sections of control nondenervated muscle subjected to double-label immunofluorescence with anti-NF70 antibodies and anti-B-50 antibodies. $A$, Phase-contrast $(P C)$ micrograph showing muscle fibers with AChE-stained endplates (arrowhead) and corresponding immunostaining of the same section with NF70 antibodies $(B)$ and B-50 antibodies $(C)$. The endplates are innervated by axon branches visualized with the NF70 antibodies. No B-50 immunoreactivity could be detected in these axon branches. $D, E$, Phase-contrast micrograph of a muscle section $18 \mathrm{~d}$ following a crush lesion $(D)$ and corresponding immunostaining with antiB-50 antibodies $(E)$. Note that the neuromuscular junctions and the intramuscular axons are heavily reactive with the anti-B-50 antibodies. $F, G$, Phasecontrast micrograph and corresponding immunolabeling with anti-B-50 antibodies of a control muscle $(F)$ and a muscle $18 \mathrm{~d}$ following crush denervation $(G)$. Only a few axons are reactive with the B-50 antibodies in the control muscle, while bright immunofluorescence is visible in reinnervating axons $18 \mathrm{~d}$ following crush.
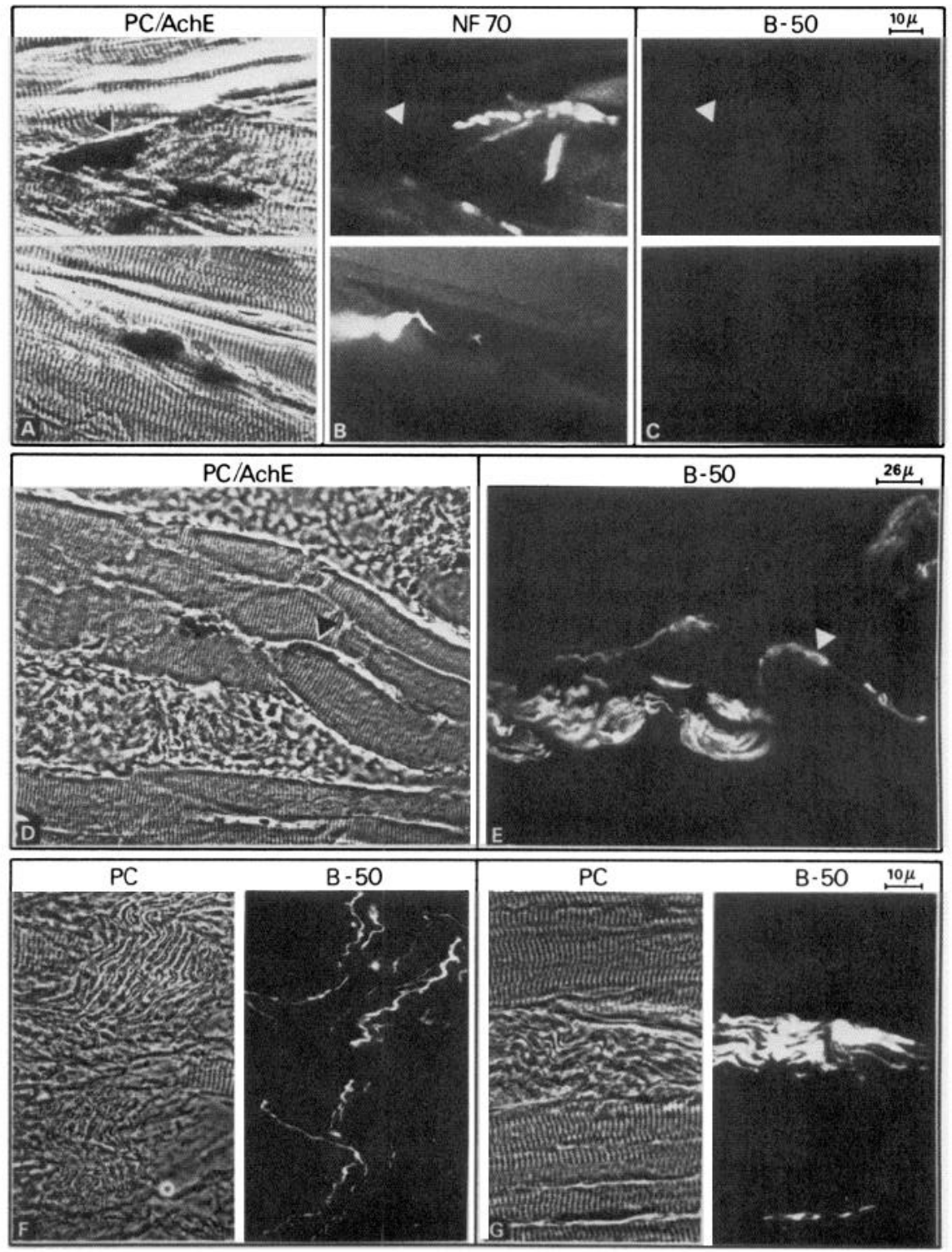

the presynaptic part of the neuromuscular junctions displayed extensive signs of degeneration. The presynaptic region of the endplate became partly occupied with Schwann cells, and vesicles, the typical presynaptic structures, were not visible in the electron microscope (Fig. 6). At this time point, diffuse immunoprecipitate was present between the invading Schwann cells in the region originally occupied by the presynaptic element of the endplate. In some instances immunoreactivity was found to be present in the superficial parts of the secondary clefts of the postsynaptic part of the neuromuscular junction (Fig. 6).

\section{Discussion}

The neuron-specific phosphoprotein B-50 has been identified in the adult brains of a variety of species, including man (Oestreicher et al., 1984). B-50 is a substrate protein of protein kinase $\mathrm{C}$, a $\mathrm{Ca}^{2+}$ - and phospholipid-dependent enzyme involved in cellular excretion and transmitter release processes (Nishizuka, 1986). The striking similarities between the properties of B-50 and a number of other proteins described in the literature led to experiments that confirmed that B-50 is identical to growth cone phosphoprotein pp46 (De Graan et al., 1985; Katz et al., 1985), protein $F_{1}$ (Chan et al., 1986; Gispen et al., 1986), and a growth-associated protein with a molecular weight of approximately $43 \mathrm{kDa}$, designated GAP43 (Skene and Willard, 1981a, b; Jacobson et al., 1986; Skene et al., 1986) or GAP48, (Benowitz et al., 1981; Benowitz and Lewis, 1983; Perrone-Bizzozero and Benowitz, 1987). Developing hippocampal and pyramidal tract neurites and isolated growth cone membranes are greatly enriched in B-50/GAP43 (De Graan et al., 1985; Katz et al., 1985; Kalil and Skene, 1986; Meiri et al., 1986; Oestreicher and Gispen, 1986). In the rat sciatic nerve, the B-50 protein was detected with radioimmunoassay and by means of immunohistochemistry (Verhaagen et al., 1986). Six days following nerve damage, a 5 -fold increase in the B-50 content per milligram of nerve protein was detected in the region of the nerve crush. Thirtysix days following the crush, B-50 levels were significantly decreased (Verhaagen et al., 1986). At this time, the outgrowing nerve fibers had reached their target cells. Hence, the drop in B-50 content in the nerve during the later stages of regeneration 

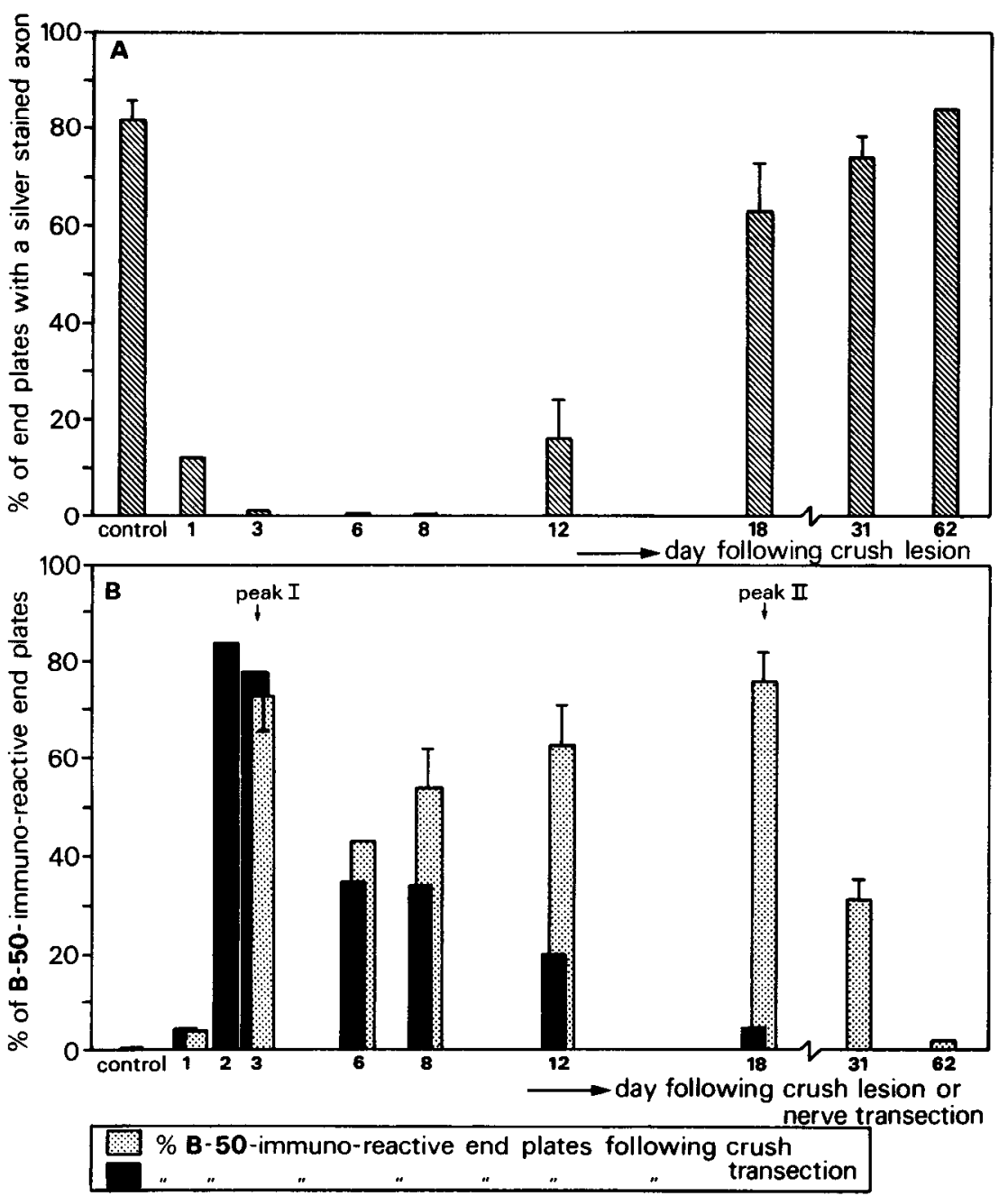

Figure 4. A, Quantification of the process of reinnervation of the soleus muscle using the AChE silver-staining procedure. Approximately 50 endplates/ muscle were screened for the presence of a silver-stained axon branch. Bars represent mean percentages $( \pm$ SEM) of endplates with an axon branch ("innervated" endplates) in sections obtained from 4 (intact, $8,12,18$, or 31 $\mathrm{d}$ following a crush) or $2(1,3,6$, or 62 d following a crush) soleus muscles. $B$, Quantification of the number of B-50immunoreactive endplates in intact and denervated muscles. The quantification procedure has been described in $\mathrm{Ma}$ terials and Methods. Bars represent the mean percentages $( \pm$ SEM) of B-50-immunoreactive endplates as determined in 4 (intact, $3,8,12$, or $31 \mathrm{~d}$ following crush denervation) or $2(1,6$, or $62 \mathrm{~d}$ following crush denervation) muscles. Following a nerve transection, the mean percentage of B-50-immunoreactive endplates in 2 muscles was determined at each time point. might be due to the disappearance of the newly formed, B-50 containing axon tips, from the region of the nerve crush. The objective of the present study was to shed more light on the fate of the B-50 protein during the process of reinnervation of muscle fibers by the regenerating peripheral nerve axons.

The time course of the reinnervation of the soleus muscle by regenerating sciatic nerve fibers was ascertained using established AChE silver-staining methods (Fig. 2). Concomitant with the period of reinnervation of the endplates in the denervated soleus muscle (8-18 d following sciatic nerve crush), a steady increase in the number of B-50-immunoreactive neuromuscular junctions became obvious (Figs. 3, 4). Subsequently the percentage of labeled endplates decreased from $80 \%$ at day 18 to $4 \%$ at day 62 after crush. Furthermore, no B-50 could be visualized in mature neuromuscular junctions (Figs. 3, 4). At 18 $\mathrm{d}$ following nerve crush, B-50 is associated with 2 types of electron-lucent synaptic vesicles with respective diameters of approximately 50 and $120-150 \mathrm{~nm}$. Most of the vesicles are of the $50 \mathrm{~nm}$ type (Fig. 5). In mature endplates, the presence of these vesicles has been correlated with cholinergic transmission. The larger vesicles constitute only a small fraction of the total vesicle population. Similar-sized vesicles have been described as transport vesicles in mature and growing axons (Brady, 1984; Mason, 1985).
In addition to the demonstration of high B-50 immunoreactivity in newly formed endplates during reinnervation of the soleus muscle, the observed presence of transient B-50 immunoreactivity in the degenerating axonal terminal is of interest and surprising, in view of the growth-association hypothesis (Skene and Willard, 1981a, b). The increase in the percentage of B-50-containing endplates during the first $2 \mathrm{~d}$ following nerve damage (Fig. 4, peak I) cannot be due to the ingrowth of sprouts into the soleus muscle because (1) the distance between the crush site and the soleus muscle is approximately $2 \mathrm{~cm}$, a distance that cannot be bridged by sprouts in $2 \mathrm{~d}$, and (2) enhanced labeling is also observed after nerve transection and ligation of the proximal nerve portion (Fig. $4 B$, peak I, solid bars). Under these conditions, sprouts cannot grow into the distal nerve stump. The results obtained with AChE silver-staining clearly show that as a consequence of the crush, all intramuscular axons undergo Wallerian degeneration. From this observation it can be concluded that the formation of collateral sprouts can be ruled out as an explanation for the early rise in the number of B-50immunoreactive neuromuscular junctions.

The electron microscope studies (Fig. 6) show that the B-50 immunoreactivity at $\mathbf{3} \mathbf{d}$ after crush is associated with degenerating nerve terminals. Corresponding to the drastic structural changes occurring in the degenerating nerve terminal, the lo- 


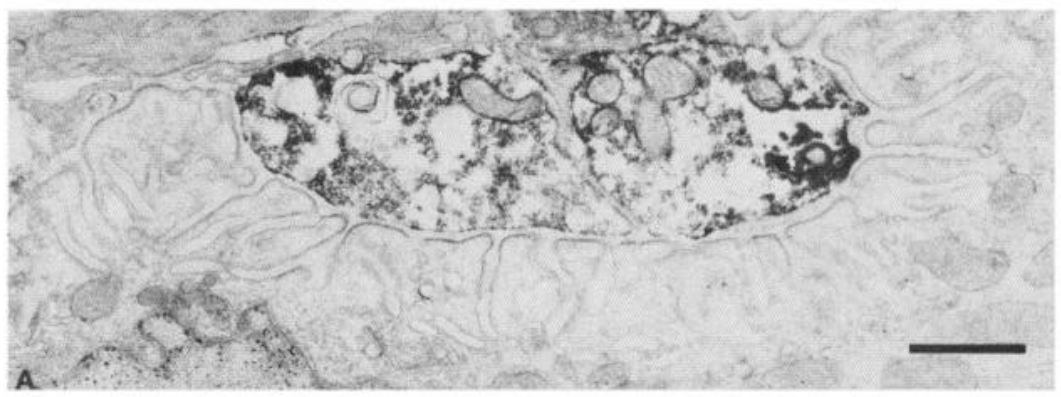

Figure 5. Electron micrographs of thin sections of newly innervated neuromuscular junctions $18 \mathrm{~d}$ following a nerve crush incubated with affinitypurified anti-B-50 antibodies. $A$, Note that the immunoprecipitate is confined to the presynaptic part of the neuromuscular junction. $B$, Larger magnification revealed labeling of presynaptic vesicles and the presynaptic plasma membranes. Bar, $1 \mu \mathrm{m}$.

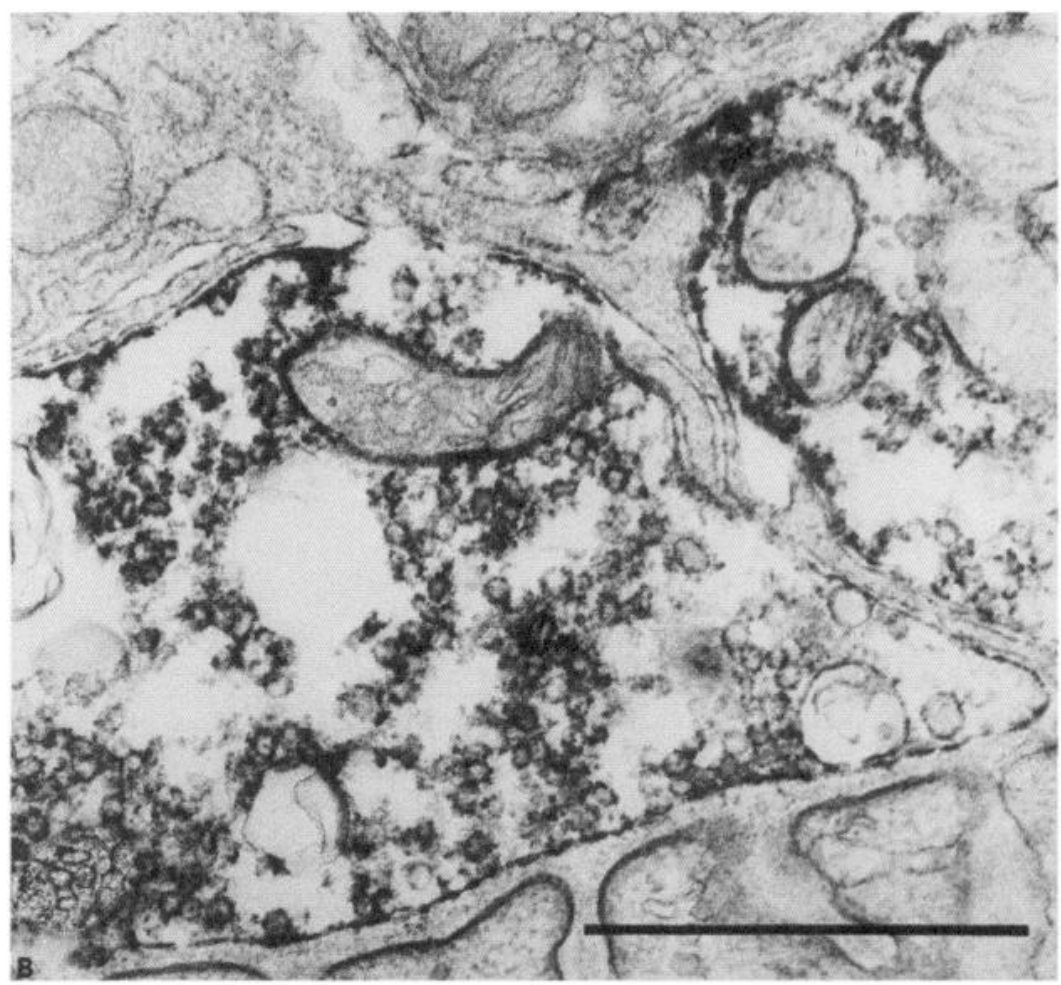

calization of B-50 immunoreactivity is disperse, perhaps because it no longer is membrane-bound.

B-50 is detectable in growing and degenerating terminals, but not in stable, mature neuromuscular junctions. A plausible explanation for this would be that B-50 is inaccessible to the antibody in mature terminals. However, treatment of sections with acetone, which permeabilizes membranes, did not alter the percentage of B-50-immunoreactive endplates in mature or newly formed terminals. This is an indication that the changes observed are not due to an immunohistochemical artifact and may be due to physiological processes occurring in the nerve. The absence of B-50 in mature neuromuscular junctions and the abundance of B-50 in sprouts that invade the denervated muscle and in newly formed neuromuscular junctions are consistent with the current concept of B-50 as a growth-associated membrane protein. The apparent increases in B-50 in degenerating terminals cannot be due to increased synthesis. However, they could result from the terminal accumulation of the low levels of B-50 originally present in the $2 \mathrm{~cm}$ nerve portion distal to the injury at the time of crush or transection. The presence of B-50 in control axons has been demonstrated (Fig. 1; Verhaagen et al., 1986), but in most axons the concentration is too low to be detected immunohistochemically. In mature, undamaged nerves, however, occasional B-50-immunoreactive axons are found (Fig. $3 F$ ). Whether the immunoreactivity derives from degeneration- or regeneration-associated changes, the appearance of B-50 in these axons may be connected with intramuscular nerve sprouting and/or synaptic remodeling. These processes have been reported to occur in the endplates of mammals throughout their life span (Wernig and Herrera, 1986).

The fact that the observed increase in B-50/GAP43 in growing, as compared to mature, nerve terminals was exactly as predicted from previous studies using different methods of investigation (e.g., the pulse-labeling studies of Skene and Willard, $1981 \mathrm{a}$, b) make it unlikely that the observations are due to artifacts. The observation that local accumulation of B-50/ GAP43 can occur in degenerating nerves, where no increased synthesis is possible, raises some important questions for further studies concerning the role of the B-50/GAP43 protein in nerve degeneration/regeneration, development, and maturation.

\section{References}

Adams, J. C. (1981) Heavy metal intensification of DAB-based HRP reaction products. J. Histochem. Cytochem. 29: 775-780.

Benowitz, L. I., and E. R. Lewis (1983) Increased transport of 44,00049,000 dalton acidic proteins during regeneration of the goldfish optic nerve: A two-dimensional gel analysis. J. Neurosci. 3: 2153-2163. 

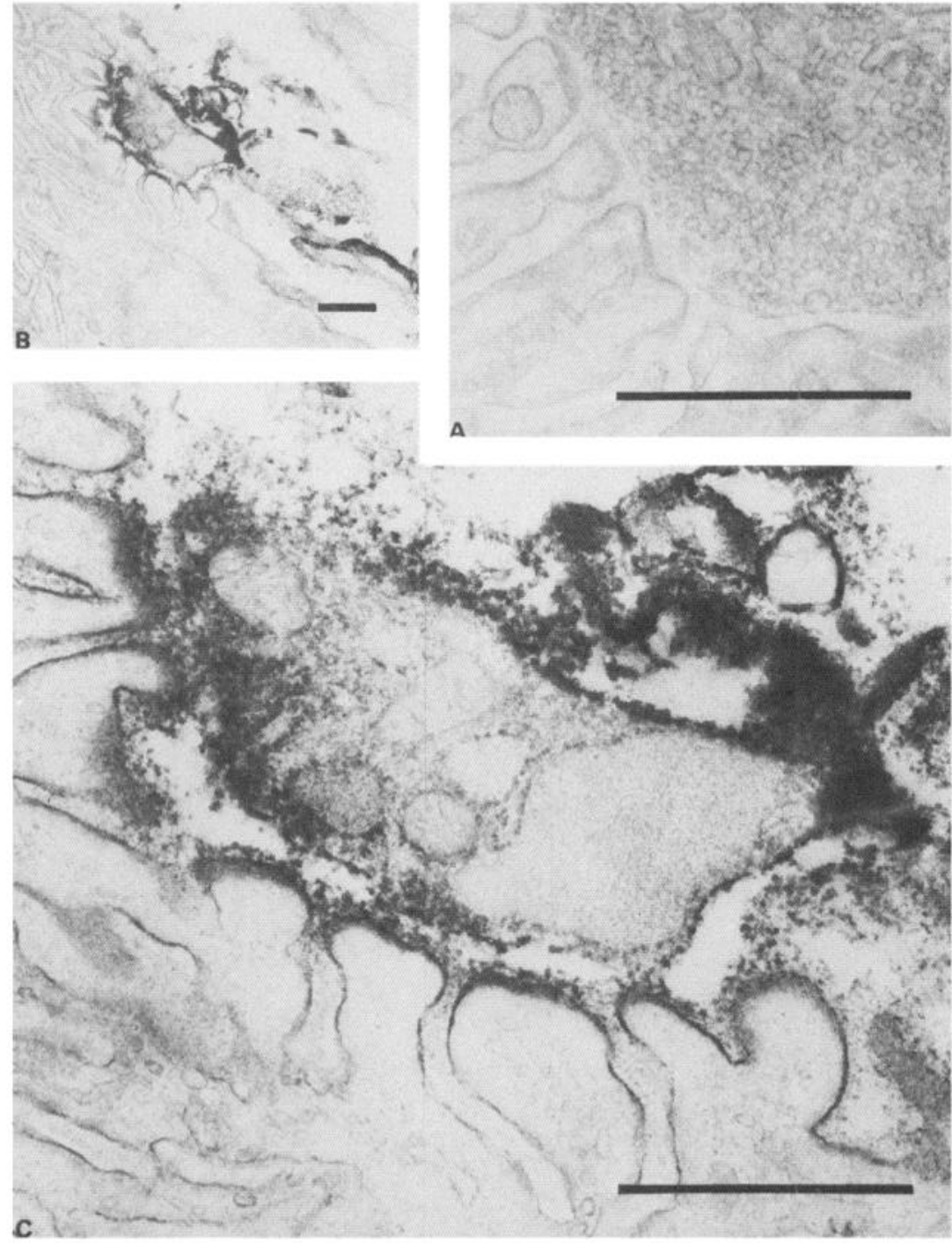

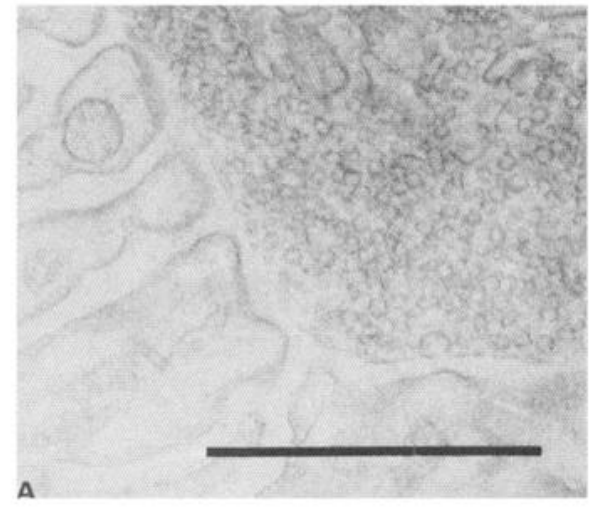

Figure 6. Electron micrographs of thin sections of $(A)$ control neuromuscular junction and of $(B, C)$ neuromuscular junction $3 \mathrm{~d}$ following crush lesion of the sciatic nerve, incubated with affinity-purified anti-B-50 antibodies. Bar, $1 \mu \mathrm{m}$.
Benowitz, L. I., M. G. Yoon, and E. R. Lewis (1981) Specific changes in rapidly transported proteins during regeneration of the goldfish optic nerve. J. Neurosci. 1: 300-307.

Brady, S. T. (1984) Basic properties of fast axonal transport and the role of fast transport in axonal growth. In Axonal Transport in Neuronal Growth and Regeneration, J. E. Elans and P. Cancelon, eds. pp. 13-28, Plenum, New York.

Chan, S. Y., K. Murakami, and A. Routtenberg (1986) Phosphoprotein $F_{1}$ : Purification and characterization of a brain kinase $C$ related to plasticity. J. Neurosci. 6: 3618-3627.

De Graan, P. N. E., C. O. M. Van Hooff, B. C. Tilly, A. B. Oestreicher, P. Schotman, and W. H. Gispen (1985) Phosphoprotein B-50 in nerve growth cones from fetal rat brain. Neurosci. Lett. 61: 235-241.

De Koning, P., J. H. Brakkee, and W. H. Gispen (1986) Methods for placing a reproducible crush in the sciatic and tibial nerve of the rat and rapid and precise testing of return of sensory function; beneficial effects of melanocortins. J. Neurol. Sci. 74: 237-256.

Gispen, W. H. (1986) Phosphoprotein B-50 and phosphoinositides in brain synaptic plasma membranes: A possible feedback relationship. Biochem. Soc. Trans. 14: 163-165.

Gispen, W. H., P. N. E. De Graan, S. Y. Chan, and A. Routtenberg (1986) Comparison between the neural acidic proteins B-50 and F1. Prog. Brain Res. 69: 383-385.

Jacobson. R. D., I. Virag, and J. H. P. Skene (1986) A protein associated with axon growth, GAP-43, is widely distributed and developmentally regulated in rat CNS. J. Neurosci. 6: 1843-1855.

Kalil, K., and J. H. P. Skene (1986) Elevated synthesis of an axonally transported protein correlates with axon outgrowth in normal and injured pyramidal tracts. J. Neurosci. 6: 2563-2570.

Karns, L. R., S. C. Ng, J. A. Freeman, and M. C. Fishman (1987) Cloning of complementary DNA for GAP43, a neuronal growthrelated protein. Science 236: 597-600.

Katz, F., L. Ellis, and K. H. Pfenninger (1985) Nerve growth cones isolated from fetal rat brain. III. Calcium-dependent phosphorylation. J. Neurosci. 5: 1402-1411.

Kristjansson, G. I., H. Zwiers, A. B. Oestreicher, and W. H. Gispen (1982) Evidence that the synaptic phosphoprotein B-50 is localized exclusively in nerve tissue. J. Neurochem. 39: 371-378.

Lowry, O. H., N. J. Rosebrough, A. L. Farr, and R. J. Randall (1951) Protein measurement with the Folin phenol reagent. J. Biol. Chem. 193: 265-275.

Mason, C. A. (1985) Growing tips of embryonic cerebellar axons in vivo. J. Neurosci. Res. 13: 55-73.

McLean, I. W., and P. K. Nakane (1974) Periodate-lysine-paraformaldehyde fixative for immuno electron microscopy. J. Histochem. Cytochem. 22: 1077-1083.

Meiri, K., K. Pfenninger, and M. Willard (1986) Growth-associated protein, GAP-43, a polypeptide that is induced when neurons extend axons, is a component of growth cones and corresponds to a major polypeptide enriched in growth cones. Proc. Natl. Acad. Sci. USA 83: 3537-3541.

Nishizuka, Y. (1986) The role of protein kinase C in cell surface signal transduction and tumour promotion. Science 233: 305-312.

Oestreicher, A. B., and W. H. Gispen (1986) Comparison of the im- 
munocytochemical distribution of the phosphoprotein B-50 in the cerebellum and hippocampus of immature and adult rat brain. Brain Res. 375: 267-279.

Oestreicher, A. B., M. van Duijn, H. Zwiers, and W. H. Gispen (1984) Crossreaction of anti-rat B-50: Characterization and isolation of a "B-50 phosphoprotein" from bovine brain. J. Neurochem. 43: 935943.

Perrone-Bizzozero, N. I., and L. I. Benowitz (1987) Expression of a $48 \mathrm{kDa}$ growth-associated protein in the goldfish retina. J. Neurochem. 48: 644-652.

Pestronk, A., and D. B. Drachman (1978) A new stain for quantitative measurements of sprouting at neuromuscular junctions. Muscle Nerve 1: 70-74.

Skene, J. H. P., and M. Willard (1981a) Changes in axonally transported proteins during regeneration in toad retinal ganglion cells. $J$. Cell Biol. 89: 86-95.

Skene, J. H. P., and M. Willard (1981a) Axonally transported proteins associated with axon growth in rabbit central and peripheral nervous systems. J. Cell Biol. 89: 86-95.

Skene, J. H. P., R. D. Jacobson, W. J. Snipes, C. B. McGuire, J. J. Norden, and J. A. Freeman (1986) A protein induced during nerve growth (GAP43) is a major component of growth-cone membranes. Science 233: 763-768.
Towbin, H., T. Staehelin, and J. Gordon (1979) Electrophoretic transfer of proteins from polyacrylamide gels to nitrocellulose sheets: Procedure and applications. Proc. Natl. Acad. Sci. USA 76: 1979-1984.

Van Hooff, C. O. M., P. N. E. De Graan, A. B. Oestreicher, and W. H. Gispen (1988) B-50 phosphorylation and polyphosphoinositide metabolism in nerve growth cone membranes. J. Neurosci. (in press).

Verhaagen, J., C. O. M. Van Hooff, P. M. Edwards, P. N. E. De Graan, A. B. Oestreicher, F. G. I. Jennekens, and W. H. Gispen (1986) The kinase $\mathrm{C}$ substrate protein B-50 and axonal regeneration. Brain Res. Bull. 17: 737-741.

Wernig, A., and A. A. Herrera (1986) Sprouting and remodelling at the nerve muscle junction. Prog. Neurobiol. 27: 251-291.

Zwiers, H., H. D. Veldhuis, P. Schotman, and W. H. Gispen (1976) ACTH, cyclic nucleotides and brain protein phosphorylation in vitro. Neurochem. Res. 1: 669-677.

Zwiers, H., P. Schotman, and W. H. Gispen (1980) Purification and some characteristics of an ACTH-sensitive kinase and its substrate protein in rat brain membranes. J. Neurochem. 34: 1689-1699.

Zwiers, H., J. Verhaagen, C. J. Van Dongen, P. N. E. De Graan, and W. H. Gispen (1985) Resolution of rat brain synaptic phosphoprotein B-50 into multiple forms by two-dimensional electrophoresis: Evidence for multi-site phosphorylation. J. Neurochem. 44: 10831090. 